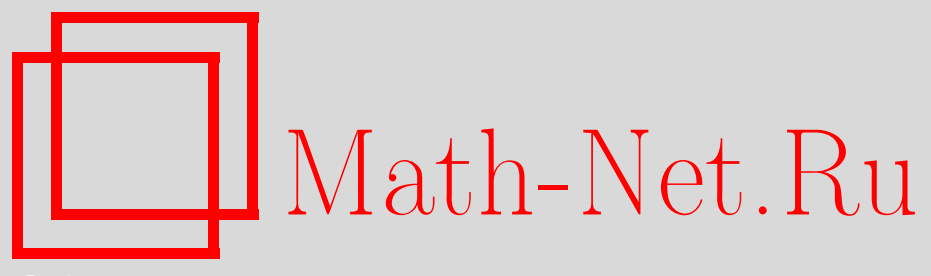

А. С. Хорошкин, Сизигии некоторых квадратичных многообразий и их связь с когомологиями алгебр Ли, УМН, 2006, том 61, выпуск 5, 189-190

DOI: https://doi.org/10.4213/rm4490

Использование Общероссийского математического портала Math-Net.Ru подразумевает, что вы прочитали и согласны с пользовательским соглашением http://www . mathnet.ru/rus/agreement

Параметры загрузки:

IP : 3.93 .64 .190

26 апреля 2023 г., 14:38:41

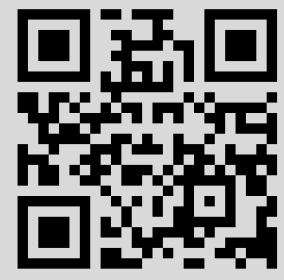




\section{Сизигии некоторых квадратичных многообразий и их связь с когомологиями алгебр Ли}

\section{А. С. Хорошкин}

1. Сизигии и когомологии алгебр Ли. Пусть $i: M \hookrightarrow \mathbb{P}(V)$ - вложение многообразия $M$ в проективное пространство, заданное набором квадратичных уравнений $Q$, так что однородное координатное кольцо $\mathscr{O}_{M}$ является квадратичной коммутативной алгеброй $S V /(Q)$. Нас интересует подсчет сизигий этого вложения, т.е. минимальный набор уравнений, минимальный набор соотношений на уравнения, минимальный набор соотношений на соотношения и т. д. На гомологическом языке этой задаче отвечает подсчет группы $\operatorname{Tor}^{S V}\left(\mathscr{O}_{M}, \mathbb{k}\right)$, где $\mathbb{k}$ - основное поле характеристики нуль. Естественная градуировка по степени на симметрической алгебре $S V$ задает градуировку на $\mathscr{O}_{M}$ и на пространствах сизигий, которую мы будем обозначать вторым индексом в $\operatorname{Tor}_{., .} S V\left(\mathscr{O}_{M}, \mathbb{k}\right)$.

У квадратичной коммутативной алгебры $\mathscr{O}_{M}$ имеется квадратично двойственная супералгебра Ли $L^{M}:=\mathscr{L} i e\left(V^{*}[1]\right) /\left(Q^{\perp}\right)$, где $V^{*}[1]$ - двойственное пространство с измененной четностью. Квадратично двойственная ассоциативная алгебра $\mathscr{O}_{M}^{!}$является универсальной обертывающей алгеброй $U\left(L^{M}\right)$. Алгебра Ли $L^{M}$ снабжена естественной градуировкой по степени элементов и содержит подалгебру Ли $L_{\geqslant 2}^{M}$, состоящую из элементов степени не меньшей 2. Имеет место следующая теорема, связывающая сизигии с этой алгеброй Ли.

Теорема [1], [2]. Если квадратичная коммутативная алгебра О्О для любой пары $(k, l)$ компонента степени $l$ в $k$-x сизигиях изоморфна компоненте степени l в $(l-k)$-x когомологиях алгебры Ли $L_{\geqslant 2}^{M}$ :

$$
\operatorname{Tor}_{k, l}^{S V}\left(\mathscr{O}_{M}, \mathbb{k}\right)=H^{l-k}\left(L_{\geqslant 2}^{M}\right)_{(l)} .
$$

Настоящая работа посвящена применению этой абстрактной теоремы к вычислению сизигий грассманиана двумерных плоскостей. Основной результат содержится в теореме 1.

2. Кривая Веронезе. Пусть $U-$ плоскость с координатами $(u, v)$. Вложение Веронезе $\mathbb{P}(U)=\mathbb{P}^{1} \hookrightarrow \mathbb{P}^{n}=\mathbb{P}\left(S^{n} U\right)$ в мономиальных координатах на $\mathbb{P}\left(S^{n} U\right)$ задается отображением

$$
(u: v) \mapsto\left(u^{n}: u^{n-1} v: u^{n-2} v^{2}: \cdots: v^{n}\right) .
$$

Соответствующая координатная алгебра $\mathscr{O}_{\text {Ver }}$ квадратичная и кошулева [3; гл. $\left.3, \S 2\right]$,

$$
\mathscr{O}_{\text {Ver }}=\mathbb{k}\left[x_{0}, \ldots, x_{n}\right] /\left(x_{i} x_{j}-x_{k} x_{m} \mid i+j=k+m\right) .
$$

В двойственных координатах $\xi_{i}$ на $V^{*}:=S^{n} U^{*}$ квадратично-двойственная алгебра Ли имеет вид

$$
L^{\mathrm{Ver}}:=\mathscr{L} i e\left(\xi_{i}\right) /\left(\sum_{i+j=k}\left[\xi_{i}, \xi_{j}\right]=0 \mid k=0,1, \ldots, 2 n\right) .
$$

Выберем фильтрацию на супералгебре Ли $L^{\mathrm{Ver}}$, упорядочив образующие следующим образом: $\xi_{0}>\xi_{n}>\xi_{n-1}>\cdots>\xi_{1}$. Старший моном $k$-го соотношения из (1) равен $\left[\xi_{0}, \xi_{k}\right]$ для $k \leqslant n$ и $\left[\xi_{n}, \xi_{k-n}\right]$ для $k \geqslant n$. Поэтому присоединенно градуированная к этой фильтрации супералгебра Ли является суммой коммутативной подалгебры Ли, порожденной $\xi_{0}, \xi_{n}$, и свободной $\mathscr{L} i e\left(\xi_{1}, \ldots, \xi_{n-1}\right)$. Известно, что любая подалгебра Ли свободной алгебры Ли свободна и имеет нулевые когомологии с номером, большим единицы. Таким образом,

$$
H^{p}\left(L_{\geqslant 2}^{\mathrm{Ver}}\right)=H^{p}\left(\mathscr{L} i e\left(\xi_{1}, \ldots, \xi_{n-1}\right)\right)=0, \quad \text { если } \quad p>1 .
$$


СледСтвиЕ $1 . \quad k$-е сизигии кривой Веронезе в $\mathbb{P}^{n}$ отличны от нуля только в степени $k+1, u \operatorname{dim} \operatorname{Tor}_{k, k+1}^{S V}\left(\mathscr{O}_{\text {Ver }}, \mathbb{k}\right)=k\left(\begin{array}{c}n \\ k+1\end{array}\right)$.

3. Грассманиан $\mathbf{G r}(2, N)$. Пусть $W-N$-мерное векторное пространство. Рассмотрим плюккерово вложение $i_{\mathbf{G r}}: \mathbf{G r}(2, N) \hookrightarrow \mathbb{P}\left(\wedge^{2} W^{*}\right)$. Естественное действие группы замены координат $\mathbf{G L}_{N}$ на пространстве $\mathbb{P}\left(\wedge^{2} W^{*}\right)$, сохраняющее $\mathbf{G r}\left(2, W^{*}\right)$, продолжается на пространства сизигий. Мы опишем это действие, используя стандартные обозначения $\pi_{\lambda}$ для неприводимого представления группы $\mathbf{G L}_{N}$, соответствующего диаграмме Юнга $\lambda$. Мы используем две формы записи для диаграмм Юнга: классическая форма $-\lambda=\left[\lambda_{1}, \ldots, \lambda_{l}\right]$, где $\lambda_{1} \geqslant \cdots \geqslant \lambda_{l}$, а также форма Фробениуса $\lambda=\left(\alpha_{1}, \ldots, \alpha_{p} \mid \beta_{1}, \ldots, \beta_{p}\right)$, где $p$ - число клеток на диагонали диаграммы $\lambda$, а значит, и ее транспонированной $\lambda^{\prime}$, числа $\alpha_{i}, \beta_{i}$ определены как длины строк и столбцов вне диагонали, т.е. $\alpha_{i}=\lambda_{i}-i$ и $\beta_{i}=\lambda_{i}^{\prime}-i$.

Определим биградуированную косокоммутативную $\mathbf{G L}_{N}$-эквивариантную алгебру $A=\bigoplus_{k, l} A_{k, l}$, где компонента $A_{k, l}$ изоморфна как $\mathbf{G} \mathbf{L}_{N}$-модуль прямой сумме представлений:

$$
A_{k, l}=\bigoplus_{\substack{N-2 \geq i_{1}>\cdots>i_{k}>1 \\ i_{1}+\cdots+i_{k}=l}} \pi_{\left(i_{1}-2, \ldots, i_{k}-2 \mid i_{1}+1, \ldots, i_{k}+1\right)} .
$$

Алгебра $A$ порождена своей первой компонентой

$$
A_{1}:=\bigoplus_{2 \leqslant l \leqslant N-2} A_{1, l}=\bigoplus_{2 \leqslant l \leqslant N-2} \pi_{(l-2 \mid l+1)},
$$

и произведение $A_{1, l_{1}} \otimes \cdots \otimes A_{1, l_{k}} \longrightarrow A_{k,\left(l_{1}+\cdots+l_{k}\right)}$ пропорционально единственному GL-эквивариантному проектору на неприводимое подпредставление:

$$
\pi_{\left(l_{1}-2, \ldots, l_{k}-2 \mid l_{1}+1, \ldots, l_{k}+1\right)} \subset \pi_{\left(l_{1}-2 \mid l_{1}+1\right)} \otimes \cdots \otimes \pi_{\left(l_{k}-2 \mid l_{k}+1\right)},
$$

если $l_{1}>\cdots>l_{k}$, и равно нулю, если какие-то из чисел $l_{i}$ совпадают.

Лемма 1. Алгебра А квадратичная и кошулева.

Теорема 1. (i) Биградуированная алгебра когомологий алгебры Ли $L_{\geqslant 2}^{\mathbf{G r}(2, N)}$ совпадает с квадратичной кошулевой алгеброй $A$.

(ii) $\operatorname{Tor}_{k, l}^{S V}\left(\mathscr{O}_{\mathbf{G r}}, \mathbb{k}\right)=A_{l-k, l}$.

Автор выражает глубокую благодарность А.Л. Городенцеву, А.С. Лосеву, М. В. Мовшеву, Г.И. Ольшанскому, Д.И. Пионтковскому, А.Н. Рудакову и Б. Л. Фейгину за ценные обсуждения

\section{Список литературы}

[1] A. Khoroshkin, Another interpretation of syzygies of Koszul algebras, Preprint ITEP-TH91/04, ИТЭФ, М., 2004; http://ellib.itep.ru/Mathphys/Preprint/Pr04.htm. [2] R. Fröberg, C. Löfwal, Homology Homotopy Appl., 4:2, part 2 (2002), 227-258. [3] A. Polishchuk, L. Positselski, Quadratic algebras, Amer. Math. Soc., Providence, RI, 2005.

А. С. Хорошкин (А. S. Khoroshkin)

Институт теоретической и экспериментальной физики E-mail: khorosh@mccme.ru
Представлено С. К. Ландо Принято редколлегией 02.08 .2006 